\title{
X-ray Quantitative Microanalysis with an Annular Silicon Drift Detector
}

\author{
Hendrix Demers ${ }^{1}$, Nicolas Brodusch ${ }^{1}$, David C. Joy ${ }^{2,3}$, Patrick Woo ${ }^{4}$ and Raynald Gauvin ${ }^{1}$ \\ 1. Department of Mining and Materials Engineering, McGill University, Montreal, Quebec, Canada. \\ 2. Center for Nanophase Materials Sciences, Oak Ridge National Laboratory, Oak Ridge, USA. \\ 3. Electron Microscopy Facility, University of Tennessee, Knoxville, USA. \\ 4. Hitachi High-Technologies Canada Inc., Toronto, Canada.
}

The scanning electron microscope (SEM) was developed for imaging applications and later, with the introduction of the $\mathrm{Si}(\mathrm{Li})$ energy dispersive spectrometry (EDS) detector, simultaneous imaging and $\mathrm{x}$ ray microanalysis became possible. However, long working distances were needed to use the EDS detector because the SEM was initially optimized for imaging only where the best spatial resolution is generally obtained at short working distance. This problem is still topical today and unfortunately $\mathrm{x}$-ray microanalysis is never performed in the best imaging conditions, i.e., not with the smallest probe size. With the introduction of the Bruker QUANTAX Annular QUAD silicon drift detector (SDD) system, scanning electron microscopy is facing a revolution. Since this detector is inserted below the pole piece. A low working distance can be used and an improved spatial resolution becomes possible. Also, since the count rate can be as high as $2000000 \mathrm{cps}$, many x-ray will be acquired in a short period of time, allowing to lower significantly the detection limit of elements and as well the minimum size of different phase features. However, the effect of the detector geometry and position on the quantification microanalysis is unknown.

Figure 1 compares the typical solid angle for SDD on the side of the chamber (with different sensor area) with an annular SDD below the pole piece. For the detector distance typical for each detector position, the solid angle for the annular detector was 10 higher. An optimum detector distance was observed at $1.5 \mathrm{~mm}$, where the solid angle was $1.35 \mathrm{sr}$. The maximum solid angle for a side SDD of 150 $\mathrm{mm}^{2}$ sensor area was only $0.09 \mathrm{sr}$ with a detector distance of $40 \mathrm{~mm}$. For quantification microanalysis where the absorption of the $\mathrm{x}$-ray is important, the value of the takeoff angle is important. Lower value increases the absorption in the sample. Also the correction model, suppose a fix value of takeoff angle. However, Figure 2 shows that the minimum and maximum of the takeoff angle on the sensor change with the detector distance. For a side detector with $80 \mathrm{~mm}^{2}$ area, the takeoff angle change from -7 to 7 degree around the center value of 30 degree. For an annular detector, the large takeoff angle $(>70$ degree) was obtained for detector distance larger than $5 \mathrm{~mm}$, which minimize the absorption effect and should give more accurate quantification results for strongly absorbed sample, for example light element analysis. At the optimum detector distance the mean takeoff angle was 33 degree with a minimum of 24 and maximum of 50 degree. The effect of this large takeoff angle variation on the correction model is currently studied.

The advantage of the larger solid angle is show in Figure 3. The detection limit $C D_{\min }$ was 10 smaller for an annular SDD. With adapted correction model, the annular SDD with is larger solid angle will clearly revolution the quantification microanalysis by moving from point analysis to quantitative micrograph with simultaneous electron imaging.

References:

[1] N. J. Zaluzec, Microscopy Today 17 (2009), pp. 56-59.

[2] R. Gauvin et al, Microscopy and Microanalysis 18 (2012), pp. 1016-1017.

[3] R. Gauvin and P. Michaud, Microscopy and Microanalysis 15 (2009), pp. 488-489. 

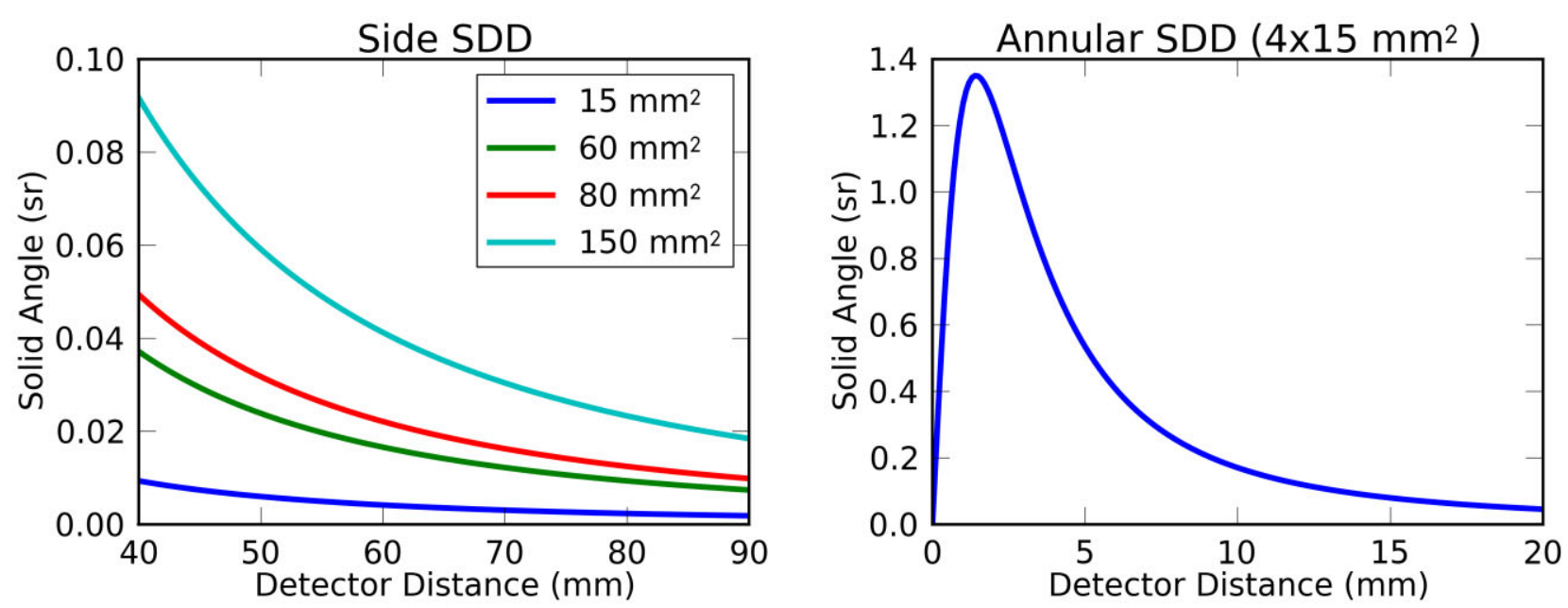

Figure 1. Variation of solid angle [1] with detector sample distance for different geometry and position of solid drift detectors (SDD). The annular SDD have four sensors.
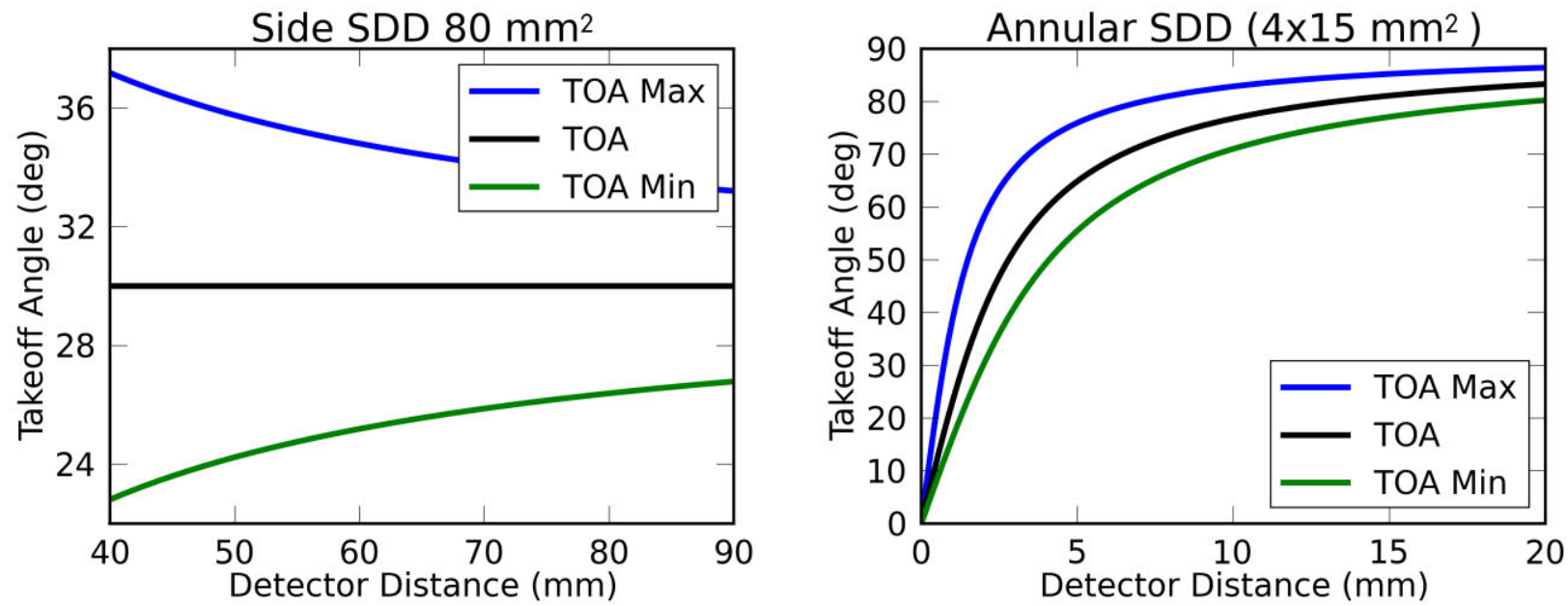

Figure 2. Variation of takeoff angle with detector-sample distance for different position of solid drift detectors (SDD).

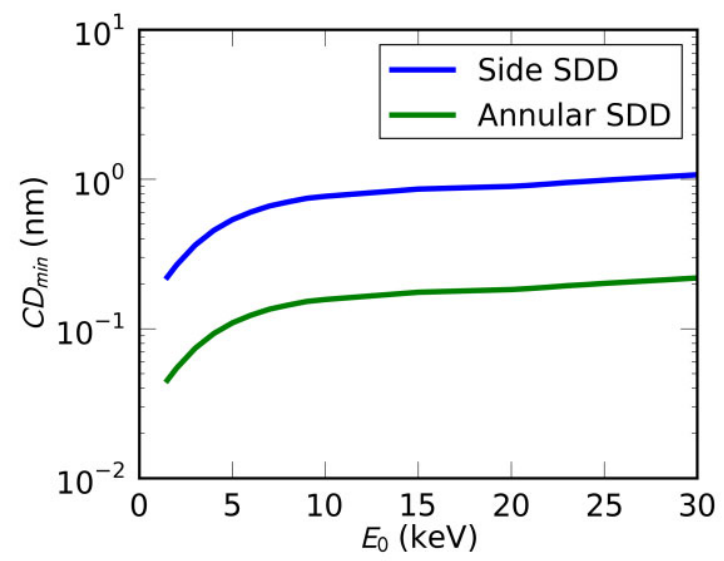

Figure 3. Variation of $\mathrm{Ti} \mathrm{L}_{\alpha}$ detection limit $C D_{\min }[2,3]$ with incident energy $E_{0}$ for different geometry and position of solid drift detector (SDD). The sample was an $\mathrm{Al}_{3} \mathrm{Zr}$ (with $1 \%$ weight fraction of Ti) nanoparticle with a diameter of $1 \mathrm{~nm}$ embedded in an Al matrix. 